of soda, rhubarb, and aromatic powder. Not infrequently cholagogues are required, and a few doses of calomel or blue pill free the patient from a tinge of jaundice, clean the tongue, and restore the appetite and digestive power.

Pleurisy and pericarditis, particularly the latter, often usher in the fatal result in cases of chronic renal disease, but much may be effected by judicious treatment. If we watch for the commencement of pericarditis, and as soon as its presence is indicated by local or abdominal pain, and by the physical signs, we apply leeches over the part, we very frequently succeed in checking the process. The same treatment is serviceable in pleurisy. The application of mustard or other counter irritants, avoiding, of course, those which irritate the kidneys, often does great good. If much effusion be poured out into the pleura one should use the aspirator, just as is done in a simple pleurisy, and that even though pericarditis coexists. I have not seen evidence that any internal medicine markedly modifies the inflammatory process. But in cases of pericarditis we should be guided by the state of the heart to the use of cardiac tonics, such as digitalis, strophanthus, or caffeine, or cardiac sedatives, such as iodide or bromide of potassium.

Increased vascular tension, whether arising comparatively early or late in the course of the disease, may be benefited by nitroglycerine or the nitrites; even small doses of nitro-glycerine often sufficing to give the most marked relief. I have not seen any injury follow its use, but it must be employed with caution in any case where there is excited cardiac action and degeneration of vessels lest extravasation of blood should occur. If such a condition exists, it is better to use the iodide or bromide of potassium, for they often improve the state of vessels, and at the same time quiet the action of the heart. Sometimes the application of cold over the heart is desirable. But far more frequently, as the case advances, degenerative changes set in in the muscular substance of the organ, and the patient is distressed by dyspnoea which may be partly uræmic, but is mainly of cardiac origin. In such a case cardiac tonics are indicated-digitalis, strophanthus, caffeine generally proving the best.

Such are, according to my experience, the most important points in connection with the treatment of chronic Bright's disease; and while they leave so much to be desired, a review of them still affords some satisfaction to those of us who are able to look back through a period of twenty or thirty years. I hope that we shall one day be able to combat the causes with a success that we do not dream of now; that we shall one day find remedies capable of directly influencing the nutrition of the kidneys, and restoring them, when diseased, to the healthy condition, or at least means of treatment, by which we may so rest the organs as to give their tissues time and opportunity of returning to the normal type. This would represent in the language of modern science what our predecessors were wont to speak of as the vis medicatrix Natura. I hope, also, that we shall find means of dealing much better than we now can with the various dangers which result from the morbid processes preventing the drain of albumen, or supplying what has been lost; securing elimination by other channels; neutralising within the system the poisons which have been produced, and, perhaps, even by a process analagous to dialysis, removing them from the system as they are formed.

\section{ON THE PRINCIPLES OF THE TREATMENT OF DIABETES MELLITUS.}

Read before the International Medical Congress, Berlin, August, 1890.

BY F. W. PAVY, M.D., LL.D., F.R.S.

THE first point to be considered in discussing the treatment of diabetes is the rationale upon which it shousd be conducted. A certain deviation from health resulting in the escape of sugar with the urine constitutes the condition that has to be combated, and something requires to be said regarding the nature of the deviation before we are in a position to approach the question of how it should be treated.

The observable phenomena are that, whilst in the healthy subject the food ingested is disposed of in such a manner within the system as not to lead to the exit of sugar from it, in the diabetic subject the food fails to be similarly disposed of, but in part passes out as unconsumed and wasted material with the urinary excretion. It is with the carbohydrate principles that the faulty action lies. These, instead of passing in the direction that results in their consumption and utilisation and thus disappearance within the system, as occurs in health, do not follow such a course, but remain in the state of carbohydrate, and are eliminated as such. The chemistry of the body with regard to these principles is at fault. The proper changes do not take place to lead to their being employed as they ought to be, and thereby lost sight of. Represented in other words, through defective assimilative action these principles do not pass on, it may be said, to their proper destination.

Thus much is learnt by simply looking at the matter through the light of ingress and egress.

There is no theoretical consideration involved in stating that the carbohydrates in the system of the diabetic fail to undergo those right chemical changes which, in health, lead to their disappearance, and that consequently, whether ingested from without, or formed from the splitting up of nitrogenous matter within, they become disposed of by egrees with the urine.

It may further, I consider, be stated that, as a result of the faulty action, the carbohydrate, in the form of sugar, reaches the general circulation in a manner it ought not. No one with any ground of support can contend that the sugar eliminated is formed by the kidney. Whatever appears in the urine has previously existed in the blood flowing to the organ, and osmosis suffices to account for the escape that takes place. I am of opinion it has satisfactorily been made out that healthy urine contains a certain small amount of sugar, and this stands in accord with what is observed as regards the condition of the blood under natural circumstances.

With regard to the presence of sugar in ordinary urine, I conducted a series of observations some years ago, in which I precipitated the sugar by means of lead acetate and ammonia, after previous separation of the uric acid by lead acetate alone. The compound of sugar and lead oxide was then decomposed by sulphuretted hydrogen, and the sugar estimated gravimetrically by boiling with the copper test liquid, collecting the precipitated cuprous oxide, and subsequently, by the aid of a galvanic current, depositing the copper upon a weighed platinum cylinder. The amount found varied from 0.096 to 0.533 parts of sugar per 1,000 parts of urine.

The condition of the blood, as regards sugar, can be with precision defined by the application of a satisfactory analytical procedure which exists at our command. There is no difficulty, with the exercise of proper attention, in securing the full extraction of whatever sugar is present in a given specimen of blood, and afterwards expressing its amount. From a large number of observations, I maystate that the quantity of sugar in blood taken under natural conditions does not amount to more than from about 0.5 to about 0.8 per 1,000 . Under deviations from the natural state the quantity may be quickly madeto rise higher, and this, it may be said, should be borne in mind in looking at results wherelarger quantities are mentioned by investigators as having been found. I have made analyses of the blood obtained from persons suffering from diabetes, and have a record of seven instances. A general agreement is distinctly recognisable between the amount of sugar escaping with the urine and that found in the blood. Taking one instance, where 750 grammes of sugar were eliminated with the urine in the twentyfour hours, the blood contained 5.763 per 1,000; whilst in another, with 27 grammes in the urine for the twenty-four hours, the amount in the blood was 1.543 per 1,000. These are the examples giving respectively the highest and lowest figures of the series, both for urine and blood. It is correct to state that the condition of the urine as regards sugar affords an index of that of the blood. This is only what might be expected, seeing that sugar is a diffusible substance, and that therefore in proportion to its presence in the blood so may it be looked for in the urine. As its presence to more than an exceedingly minute extent is abnormal to the urine, so the same may be said of the blood, and its presence in the blood to the extent occurring in diabetes means the existence of an unnatural state of this fluid, which induces a deviation from healthy action throughout the system. In proportion to the extent of this deviation from the healthy state-that is, in proportion to the amount of sugar reaching the general circulation and thence passing out through the kidney-so will stand the measure of severity of the symptoms of diabetes. Looked at broadly, it may certainly be stated that the larger the amount of ouger elimingted with the urine the worse, in every direction, is the condition of the patient suffering from diabetes. 
We thus trace the symptomatic phenomena of the disease to the abnormal condition occasioned by the presence to an undue extent of sugar in the general circulation.

Whence, it may be next asked. arises this abnormality? I must not enter too far into the discussion of this matter, but the question has a distinct bearing upon the basis of treatment, and therefore requires to be touched upon to a certain extent.

I doubt not it will be conceded by all that the object to be attained by treatment is to diminish the deviation from health as far as practicable. It is only a rational procedure to endeavour to establish and maintain as close an approximation to the healthy standard as our knowledge enables us to effect. Observation shows that the amount of error as regards sugar in the blood, and, following upon this, sugar to be discharged with the urine, is in proportion to the amount of carbohydrate principles, of whatever kind, ingested. It may be said in general terms without, as I have already stated, asserting anything outside the region of fact, that the nature of the error to be dealt with consists in a failurd of the power in the system to dispose of the carbohydrates in a manner to lead to their utilisation and disappearance. But now arises the question, to what kind of faulty action is this failure to be attributed? Two points of view present themselves for consideration. We start with the fact that sugar is present in the blood to an extent that is unnatural. Is this due to sugar reaching the general circulation in a manner that it ought not? Or is it to be regarded as natural that all the sugar eliminated in diabetes should reach the general circulation, the error consisting of its not undergoing subsequent destruction, thus leading to accumulation?

It would be out of place to discuss these propositions here. The view to be taken rests on physiological considerations. It is known that I have over a long space of time given close attention to the matter, and my experinental inquiries lead me decidedly to affirm that $I$ consider the source of the sugar encountered in the blood and eliminated in diabetes to be attributed to its being permitted to enter the general circulation in a manner that it ought not. This view harmonises fully with the phenomena observed in diabetes. In health I should say the opportunity is not afforded for the ingested carbohydrates to appear in the urine for the reason that they are not permitted to pass through the liver and reach the general circulation. In diabetes, on the other hand, we know that they do reach the general circulation in the form of sugar, and from the amount of this principle to be found in the urine it can be stated that they must do so in proportion to the amount ingested. I would, therefore, say that we have here to deal with a failure of power-assimilative, or whatever else it may be called-to arrest the passage of carbohydrates through the liver. Being thus permitted to reach the general circulation, they are placed in a position to be discharged with the urine, and hence, according to the amount of carbohydrate principles ingested, so is the amount of sugar eliminated. With such a state of things existing, elimination necessarily follows upon, and is proportionate to, ingsstion, and leads to the production of a result which is found to stand in harmony with observation.

I have spoken of ingested carbohydrate being checked by the liver from entering the general circulation as constituting what occurs under conditions of health, and I do not make this statement unsupported by the information afforded by experiment. I have conducted a large number of experiments upon the point, and can say from them that when the requisite precautions are observed, to obtain a representation of the natural condition of the blood of the systemic or general and of the portal circulation, a large preponderance of sugar is encountered in the blood of the portal vein, if the observation be made at a period of digestion and after the ingestion of food freely containing carbohydrate matter. But it is necessary to bear in mind, in the estimation of the sugar be effected, as is the common practice, with the copper test, that a fallacy may arise from the following circumstance, unless measures are taken to guard against it. With the transformation of starch in the alimentary canal, preparatory to absorption, it is not, certainly to any noteworthy extent, carried higher than maltose, which, as is known, has a cupric oxide reducing capacity of 61 , as compared with glucose at 100 , and much of it is only carried into a dextrin with a lower cupric oxide reducing power still. Hence the form of carbohydrate, derived from starch which reaches the portal system, does not possess the cupric oxide reducing capacity of glucose, but something more or less considerably below it. As an actual fact I have a recorded instance in which the product contained in the portal blood after the inges- tion of starchy food possessed a cupric oxide reducing power standing as low as 21 , as compared with glucose at 100 . In this instance, if reliance had been placed upon the ordinarily conducted method of estimation, the amount of carbohydrate present would have been expressed at only about one-fifth of what it really was. With a form of carbohydrate other than glucose exisiting, it is necessary to bring it into glucose by boiling with dilute sulphuric acid, to permit of the true amount being determined, and this has been the plan of procedure of late years adopted in the researches I have conducted.

I have considered it necessary to enter into these preliminary details. They display the nature of the faulty condition that has to be dealt with by treatment. Sugar reaches the general circulation in a manner that it ought not, and to its presence in the system are due the various symptoms belonging to diabetes. Through reaching the general circulation it becomes eliminated by the kidney and is lost. The disease thus involves a sacrifice of material which ought by rights to be turned to account, but this is a point that has but little bearing on the production of the phenomens that are observed in connection with the disease. If it were only a question of waste of the carbohydrate principles of food there would be no reason against their being taken and allowed to run off. Provided a sufficient amount of other alimentary principles were consumed to meet the requirements of life no particular harm need arise from the sacrifice of the material occurring. What, it may be said, in reality inflicts the harm is the altered constitution of the blood, occasioned by the presence in it of the sugar which passes through the system to the urine. In proportion to the largeness of the amount of sugar thus traversing the system in the blood so will be the extent of deviation from the natural state and so in correspondence the impairment of health that will be found to exist.

The class of case to which these remarks apply is that in which the discharge of sugar is susceptible of control by treatment, and the class embraces the majority of the cases in which the disease sets in after the middle period of life.

In such instances, starting with the ingestion of carbohydrate, there follows, briefly summarised, as a consequence of the want of proper transformative or assimilative power within the system, an accumulation of sugar in the hlood attended with its discharge by the urine. Accumulation of sugar in the blood leads to the production of symptoms proportionate in severity to the deviation from the natural state. The plain object before us is to reduce this deviation as far as is found to be possible.

We cannot be wrong in endeavouring to attain as close an approach to natural conditions as circumstances permit. If the chemistry could be set right, and sugar be prevented reaching the general circulation, the disease would be removed; but it may not be possible to restore the transformative or assimilative power which has become impaired or lost, and then the only way of arriving at what is wanted is to withhold from introduction into the system the alimentary principles which, owing to failure of power to properly dispose of them, cannot be of service, and which, by leading to the passage of sugar through the system, establish an unnatural condition and thereby inflict positive harm.

As long as the passage of sugar through the system is prevented no harm takes place. In the course of all my experience in diabetes I have never known anything serious to arise as a part of the disease so long as the urine has been kept free from sugar. There is nothing, in fact, to form the source of trouble, seeing that there is not the abnormal presence of sugar in the circulation to occasion devistion from the healthy state. On the other hand, when sugar is passing through the system, and the remark applies in proportion to the amount passing through, not only are there to be observed the symptoms ordinarily consequent thereon, but a constant state of insecurity exists, from the danger of the supervention of the serious issues known to follow upon disease. Moreover, with the unnatural state occasioned by the presence of sugar, nutritive action is not carried on in such a manner as to properly maintain the general strength. As a consequence, the general power becomes sapped or prematurely exhausted, and the system weakened and rendered less able to resist the effect of pernicious influences. Such is not the position when sugar is not similarly traversing the system. Indeed there is nothing to render the state essentially different from that ordinarily existing.

The contrast between the two conditions-that is, where sugar is allowed to abnormally exist in the system, and where it is pre- 
vented from doing so-is well shown in cases where the disease has run on for some time without being recognised, and is subsequently controlled by dietetic treatment. What will be observed in such instances will be a gradually advancing impairment of health and increasing severity of the symptoms of the disease and it is right to assume that progress in the same direction would run on, and the patient grow worse and worse, if the condition continued to be left to itself. Whilst matters are thus proceeding, it happens, say, that the existence of the disease becomes recognised, and, if the case be such that the sugar is susceptible of being removed from the urine by the exclusion of the carbohydrate principles from the food, and this exclusion be carried out, this alone will suffice, not only to check the downward progress occurring, but to bring back health and strength to the patient.

The first consideration, therefore, in the treatment is to control by dietetic measures the passage of sugar through the system. The real point, however, to be aimed at is to restore the assimilative power over the carbohydrate elements of food; and until this has been accomplished it cannot be said that a cure has been effected, but only that the disease is held in subjection, and prevented, as long as the condition can be maintained, from leading on to an unfavourable issue. What most conduces to this desired restoration of assimilative power is the maintenance of a normal state of the system by keeping it free from the passage of sugar through it, and in this way bringing a healthy condition of body to bear in helping to promote a removal of the faulty state.

According to my own experience, opium and its derivatives, codeine and morphine, are the medicinal agents which, more than any others that I know of, assist in the actual cure of the disease, by which I mean a restoration of the assimilative power which has been impaired.

The influence of these agents may be witnessed in cases where the sugar has been brought down by diet to a certain point, but is insusceptible of entire removal from the system by dietetic treatment alone. The complete removal may then be sometimes observed to follow the subsequent administration of the drug showing that the medicinal agent has acted in the direction of exerting a restraining influence over the abnormal production and elimination of sugar.

When cases of a favourable nature, that is, cases occurring above the middle period of life, are treated by these combined measures, and the treatment is steadily carried on for some time, it is a matter of common observation that the system of the patient becomes able to tolerate a certain amount of carbohydrate food, without it leading to the elimination of sugar. Often, with strict observance of the required treatment, the assimilative power is found to become so far re-established, that a fair amount of the carbohydrate principles, or even an ordinary diet, may be taken without leading to the elimination of sugar. When this is the case, carbohydrate principles, according to the extent found to be tolerated, may be taken without occasioning harm; but the object is to keep below the point at which the escape of sugar takes place, and when this is done actual benefit, instead of injury, is derived therefrom.

Here I may refer to the aid afforded by the quantitative testing of the urine. It is absolutely essential, I consider, in the management of a care, to possess the knowledge thus supplied, not only for the purpose of regulating the treatment according to the progress made, but also for keeping a check upon the manner in which the directions given are being carried out. When in a case it is found to happen that the assimilative power has been restored, it is permissible to consider that an actual cure has been effected; but it is always requisite to bear in mind that a weak point has existed, and that it is advisable to avoid unduly taxing a power which has previously given evidence of being at fault.

Haavy Damages.-In the Nisi Prius Court at the Birmingham Assizes on Wednesday, August 13th, the case of Mountney $v$. Moxon was tried before Mr. Justice Hawkins. The plaintiff is the son of a farmer at Snitterton, Derbyshire, and the defendant is a medical practitioner at the same place. According to the newspaper report which has reached us, in April last plaintiff broke his leg during a football match, and was professionally attended, first by the defendant's assistant, named Rains, and afterwards by the defendant himself. It was alleged that through unskilful treatment mortification set in, and amputation of the foot was necessary. Plaintiff claimed $\mathbf{£} 4,500$. After the case had been part heard, a compromise was effected, it being understood that the plaintiff had accepted $£ 450$ as compensation.

\section{BRITISH MEDICAL ASSOCIATION. SUBSCRIPTIONS FOR 1890.}

SUbscriptions to the Association for 1890 became due on January 1st. Members of Branches are requested to pay the same to their respective Secretaries. Members of the Association not belonging to Branches are requested to forward their remittances to the General Secretary, 429, Strand, London. Post-office orders should be made payable at the West Central District Office, High Holborn.

\section{Ibe Britígh attedical Tournal.}

\section{SATURDAY, AUGUST 16itH, 1890.}

\section{THE INTERNATIONAL CONGRESS, BERLIN.}

Congressus haud impar-Berlin has had to sustain the stress of a gathering of medical men of all nations, in numbers hitherto unprecedented. They invaded the metropolis of the German Empire in hosts from every part of the world. They came ; they saw ; and they admired. Nothing could have exceeded the overflowing hospitality, the generous and cordial welcome, the thoughtful courtesy, and the minutely elaborate preparations with which the social and scientific needs of the invading hosts were met. All were alike made to feel this welcome ; so that whether for its remarkable assemblage of the most eminent representatives of cosmopolitan medical science or for its endless round of scientific and social activity, the Berlin Congress must be pronounced to have far exceeded any which has preceded it; and it will certainly be difficult in the future for any capital to surpass the feat which Berlin has ascomplished.

The Presidents of the Sections and the Berlin Professors vied with each other in their devotion to the work of the week. Their attention to the business of the Sections, their courtesy to their guests, their munificent hospitalities could not be surpassed. Professor Virchow was ubiquitous, untiring, and ever smiling - amiable to all, and heedless of nothing which could contribute to the success of the meeting-while all did their work in a way which must leave an ineffaceable feeling of friendship and thankfulness on the minds of those whom they came in contact. There were some prominent men -among whom were Professor von Bergmann, Professor Leyden, Professor Oscar Liebreich-who seemed to have more than ordinary power of guiding, and whose courtesies and activities were the theme of universal comment. The work of Dr. Guttmann-the able editor of the Deutsche medizinische Wochenschrift-who presided over the literary arrangements of the Congress, occupied him frequently all through the night as well as throughout the day. Dr. Ewald, the editor of the Berliner klinische Wochenschrift, was less overworked, but not less indefatigable and successful in the exercise of his social and scientific energies. But where so many contrived to do each one the work of a dozen, and where all fulfilled their functions with the utmost energy and devotion, it is almost invidious to single out any individuals for special thanks.

The labours of Dr. Lassar, the General Secretary, and the chief officers of the Congress were arduous and incessant. This will be easily understood when it is remembered that upwards of 6,000 persons had to be pro- 